\title{
Chemical Separation of Fixed Tissue Using Thermolysin
}

\author{
Anahita Dua ${ }^{1}$ and Sapan S. Desai ${ }^{2}$ \\ ${ }^{1}$ Center for Translational Injury Research (CeTIR), Department of Surgery, University of Texas-Houston, 6413 Fannin Street, \\ Houston, TX 77034, USA \\ ${ }^{2}$ Department of Surgery, Duke University, Durham, NC 27707, USA
}

Correspondence should be addressed to Sapan S. Desai; sapan.desai@surgisphere.com

Received 12 June 2013; Revised 16 July 2013; Accepted 16 July 2013

Academic Editor: Francesco Cappello

Copyright (C) 2013 A. Dua and S. S. Desai. This is an open access article distributed under the Creative Commons Attribution License, which permits unrestricted use, distribution, and reproduction in any medium, provided the original work is properly cited.

\begin{abstract}
Thermolysin is a metallopeptidase used to cleave peptide bonds at specific junctions. It has previously been used to cleave specific amino acid sequences found at the junction of the sensory epithelium and underlying stroma of unfixed otolithic organs of the vestibular system. We have used thermolysin to separate sensory epithelium from the underlying stroma in fixed cristae ampullares of mouse, rat, gerbil, guinea pig, chinchilla, and tree squirrel, thus removing the saddle-shaped curvature of the sensory organ and creating a flattened sensory epithelium preparation. This permits visualization of the entire sensory organ in a single mount and facilitates proper morphometric analysis.
\end{abstract}

\section{Introduction}

Thermolysin, otherwise known as protease type $\mathrm{X}$, is found in Bacillus thermoproteolyticus [1]. Thermolysin is a metallopeptidase and cleaves peptide bonds at the N-terminal of hydrophobic amino acids including alanine, isoleucine, leucine, methionine, phenylalanine, threonine, tryptophan, tyrosine, and valine [2]. Thermolysin was first characterized by Matsubara et al. [3] and subsequently used in unfixed otolith organ tissue to cleave specific amino acid sequences found at the junction of the sensory epithelium and underlying stroma [3, 4]. Suzuki et al. [4] were able to use thermolysin to cleave proteins anchoring the otolithic membrane to the tectorial membrane [4]. After centrifugation, they were able to separate the gelatinous layer from the otolithic stones. Saffer et al. [5] used thermolysin to separate the sensory epithelium from nonsensory epithelium in unfixed utricular maculae of rats [5]. Thermolysin has also been used to separate unfixed human keratinocytes, cultured intestinal epithelial cells, cultured cochlear spiral ganglion neurons, and pancreatic islet cells [6-10]

Thermolysin has not previously been used to separate fixed tissue, and there is concern that the use of fixatives such as glutaraldehyde, formaldehyde, and acrolein may change the protein structure such that it is no longer amenable to cleavage by thermolysin. Despite this concern, we have used thermolysin to separate sensory epithelium from the underlying stroma in fixed cristae ampullares of mouse, rat, gerbil, guinea pig, chinchilla, and tree squirrel (Figure 1). This resulted in removal of the extracellular matrix that imparts the three-dimensional saddle shape of the sensory organ and the creation of a flattened sensory epithelium preparation. This two-dimensional view was desired to improve visualization of the entire sensory epithelium at once and to allow more accurate morphometric analysis.

The shape of the cristae has made them resistant to experimental methods that rely on surface preparations and to microscopic analysis due to depth of field limits. This issue is especially significant in immunochemistry, where it is sometimes necessary to have a flat preparation in order to best visualize the specimen. In a section from a crista that has not been treated with thermolysin, the central zone is nearly at a right angle to the peripheral zone. Mapping the location and type of specially stained calyx afferents becomes impractical due to this curvature, especially when using intact preparations. Using camera lucida to create this map fails due to the horizon effect: calyx afferents overlap each other in the map while focusing through the sample due to the curvature 


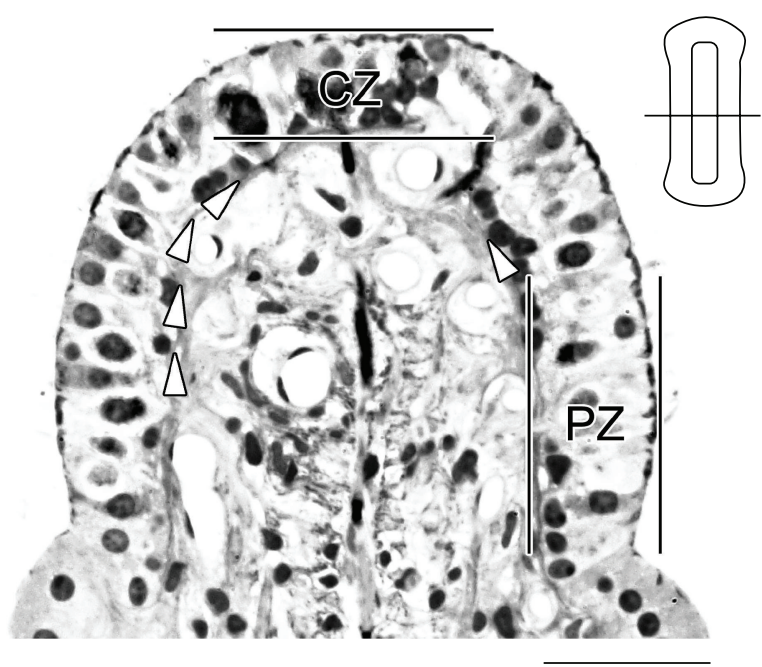

(a)

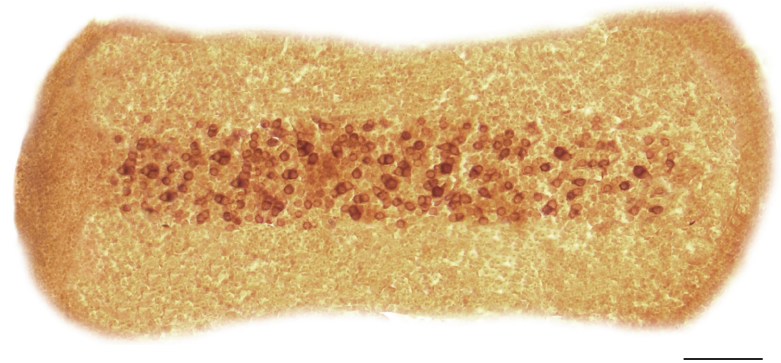

(b)

Figure 1: Photographs illustrating the significant orthogonal projection of a section of crista ampullaris and the benefits of enzymatically flattening the sensory epithelium. (a) A transverse section from a crista ampullaris showing the significant three-dimensional projection of the sensory epithelium. The central zone (CZ) is oriented such that it is nearly at right angles to the peripheral zone (PZ). When viewed from above, it is difficult to create a reconstruction due to one of these parts continuously being out of the focus. Thermolysin solves this problem by enzymatically cleaving the tissue at the junction of the sensory epithelium and stroma, indicated by the white arrow heads. The inset shows the relative location of the transverse section from a standard reconstruction of a crista. Scale bar $=50 \mu \mathrm{m}$. (b) A flattened crista ampullaris from a chinchilla stained with calretinin antibody. Scale bar $=100 \mu \mathrm{m}$.

of the crista. A flat preparation is necessary to remove the orthogonal projection of the crista.

\section{Methods}

2.1. Animals. Horizontal and vertical cristae were obtained from male and female wild-type adults from six species representing the three suborders of Rodentia myomorpha: mouse, rat, and gerbil; Caviomorpha: guinea pig and chinchilla; and Sciuromorpha: tree squirrel. For all species except squirrels, animals were anesthetized using sodium pentobarbital $(80 \mathrm{mg} / \mathrm{kg}$, i.p.). Tree squirrels were cage-trapped in the wild (IL Dept of Natural Resources Scientific Permit number W390) and anesthetized with inhaled $4 \%$ isofluorane. Each animal was perfused transcardially with heparinized $(1000 \mathrm{IU} / 100 \mathrm{~mL})$ isotonic vascular rinse until the perfusate was clear of blood (about one min), followed by $10 \mathrm{~min}$ of fixative (4\% paraformaldehyde, $1 \%$ picric acid, and $1 \%$ acrolein) made in $0.1 \mathrm{M}$ phosphate buffer (PB). Following perfusion, temporal bones were immersed in the same fixative for $20 \mathrm{~min}$. Sensory organs were dissected in PB and processed en toto sequentially through $4 \%$ Triton X-100 (1 hr), Cal-Ex decalcifying solution (Fisher Scientific, Pittsburgh, PA, USA, $10 \mathrm{~min}$ ), and $1 \%$ sodium borohydride (10 min) with $0.1 \mathrm{M} \mathrm{PB}$ rinses between treatments. All animal tissue was harvested in accordance with the University of Illinois at Chicago Institutional Animal Care and Use Committee (IACUC) approved protocols.

2.2. Immunohistochemistry. Whole sensory organs were blocked in $10 \%$ normal goat serum (Chemicon, Temecula,
CA, USA) in $0.01 \mathrm{M}$ phosphate buffered saline (PBS) for $1 \mathrm{hr}$, followed by 1:1000 rabbit anti-guinea pig calretinin IgG (Chemicon) in blocking solution (18-24 hrs), and 1:1000 biotinylated goat anti-rabbit IgG (Chemicon) secondary antibody in PBS (2 hrs). Sensory organs were rinsed, incubated per ABC Elite kit instructions (Vector Laboratories, Burlingame, CA, USA) for two hrs at room temperature or overnight at $4^{\circ} \mathrm{C}$, and reacted using $0.5 \%$ DAB (Sigma, St. Louis, MO, USA) and $0.003 \%$ hydrogen peroxide in $\mathrm{PB}$.

2.3. Flattening Procedure. All cristae were stored in $4 \%$ paraformaldehyde in $0.1 \mathrm{M}$ phosphate buffer for approximately 2 years while awaiting further analysis. When we were ready to study the cristae, they were removed from the fixative and rinsed in $0.1 \mathrm{M}$ phosphate buffer. Samples were then incubated with $1 \mu \mathrm{g}$ thermolysin (Sigma Chemical Co., St. Louis, MO, USA), $2 \mathrm{mM}$ Tris- $\mathrm{HCl}$, and $1 \mathrm{mM} \mathrm{CaCl}_{2}$ in a total volume of $50 \mathrm{~mL} \mathrm{H}_{2} \mathrm{O}$ at $55^{\circ} \mathrm{C}$ in a covered beaker for 12-18 hours. Samples were then rinsed in $0.1 \mathrm{M}$ phosphate buffer, and if the sensory epithelium was not already floating at the top of the solution, it was easily microdissected from the underlying stroma using a pair of fine forceps. The sensory epithelium was adhered to a slide, coverslipped using glycerol, and prepared for photography and calyx afferent counts.

Additional cristae from recently perfused animals were also flattened using thermolysin. These cristae were incubated in thermolysin immediately after they were reacted using $\mathrm{ABC}$ and DAB. The protocol used was similar to that described previously, except the incubation time was 18 hours and $2 \mu \mathrm{g}$ of thermolysin were used. Sensory organs were then 
photographed and digitized for quantitative morphometry. These morphometric measurements are not corrected for shrinkage artifact, which we estimated to range from 5$10 \%$. Camera lucida drawings were made of the labeled calyx afferents in each sensory organ.

\section{Results}

We used thermolysin in tissue perfused with paraformaldehyde. The relative ease of separation of the sensory epithelium from the surrounding transitional epithelium and underlying stroma indicates that the protein cross-links formed by the fixative do not compromise the peptide bond cleavage activity of thermolysin. We performed our sensory epithelium separations on tissue stored in $4 \%$ paraformaldehyde for more than 2 years-it is clear that this particular fixative had no effect on thermolysin function.

We also used thermolysin on recently fixed tissue where the sensory organs were incubated in thermolysin immediately after they were reacted with $A B C / D A B$. In order to achieve the same results as tissue fixed for longer periods, we had to double the concentration of thermolysin (from $1 \mu \mathrm{g}$ to $2 \mu \mathrm{g}$ ) and incubate the tissue at $55^{\circ} \mathrm{C}$ for 18 hours. We believe that the longer incubation time is necessary for fresh tissue because the protein bonds between the stroma and sensory epithelium have not had time to weaken, as it may have even in tissue that had been in fixative for two years.

In a section from a crista that had not been treated with thermolysin, the central zone is nearly at right angles to the peripheral zone (Figure 1(a)). After the use of thermolysin, a flat preparation can be created to permit more accurate morphometric analysis (Figure 1(b)). Digestion of the peptide layer connecting the otolithic membrane to the underlying sensory epithelium was also completed using thermolysin (Figure 2). The sensory epithelia of the utricular and saccular maculae were successfully prepared using thermolysin, permitting subsequent camera lucida drawings of the now exposed mechanosensory hair cells that could be identified through the use of immunohistochemistry techniques (Figure 3). There is no breakdown of the sensory epithelium itself, including the overlying mechanosensory hair cells.

\section{Discussion}

We have demonstrated that thermolysin can be used to separate the sensory epithelium from the underlying stroma and surrounding transitional epithelium in fixed tissue. Thermolysin is likely cleaving peptide bonds found in certain hydrophobic amino acids located at these junctions. Preparing flattened sensory epithelium specimens from fixed tissue allows us to perform calyx afferent counts with accuracy and precision that would otherwise be inaccessible. Using thermolysin in preparations with fixed tissue, such as with keratinocytes, intestinal epithelial cells, spiral ganglion neurons, and otoconial membranes, may be feasible.

It has been suggested that thermolysin cleaves certain hydrophobic amino acids found in glycosaminoglycans (GAGs) [11]. Wislocki and Ladman [12] in 1955 demonstrated

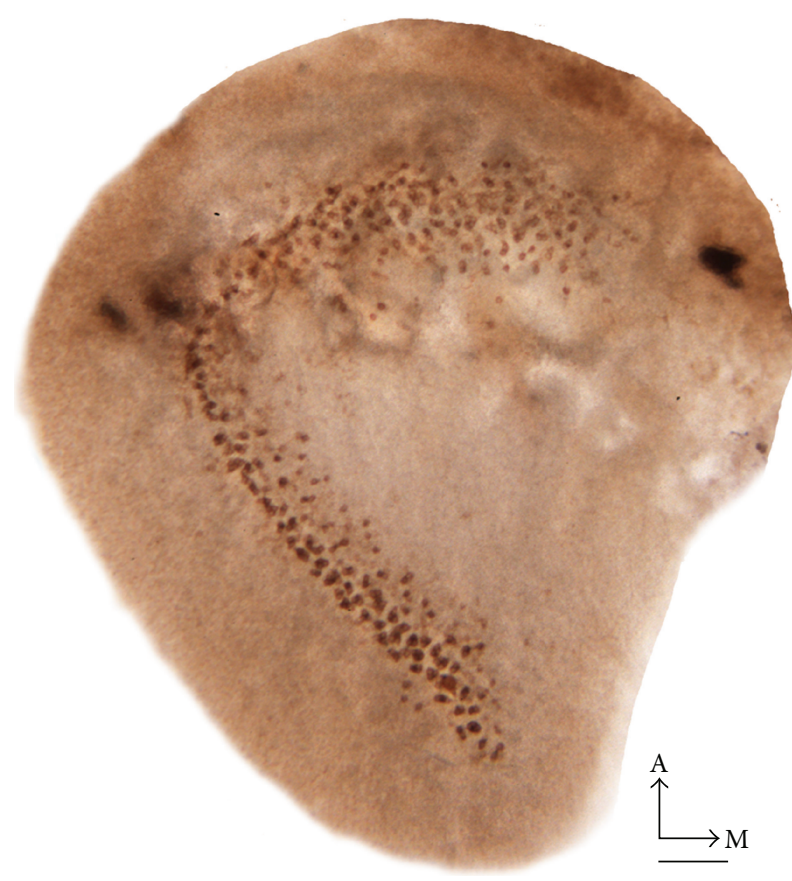

Figure 2: Photomicrograph of chinchilla utricular macula showing calretinin antibody-labeled calyx afferents. The peptide bonds connecting the otolithic membrane to the underlying sensory epithelium have been digested through the use of thermolysin, allowing immunohistochemistry techniques to localize the mechanosensory hair cells. Scale bar $=100 \mu \mathrm{m}$.

the presence of GAGs in the otoconial membrane using a periodic acid-Schiff reaction. Others have indicated the presence of sulfated GAGs in the otoconial membrane using radiography [13-15]. Recently, keratin sulfate immunoreactivity in otoconial membranes was demonstrated using monoclonal antibodies [16]. Suzuki et al. [11] have found uronic acids, which are essential components of most GAGs, in the otoconial membrane [11].

Though it is likely that thermolysin cleaves peptide bonds found in the amino acids of otoconial membrane GAGs, the target of thermolysin action between the sensory epithelium and stroma is unknown. Hultcrantz and Bagger-Sjöbäck [16] have demonstrated the presence of chondroitin-4-sulfate in sensory hair cells and within the subepithelial layer. Keratan sulfate was found in the lining of vestibular hair cells and in nerve tissue [16]. Thermolysin cleaves peptide bonds within these two GAGs and other proteins to weaken points of contact between the sensory epithelium and stroma.

The presence of keratan sulfate in the nerve tissue explains why prolonged treatment with thermolysin leads to a general breakdown of the nerve epithelium. During separation of the sensory epithelium from the underlying stroma after thermolysin treatment, it is not uncommon for parts of the nerve tissue to break off. Pulling on these nerve fibers is often used to our advantage to help separate the sensory epithelium from the transitional epithelium. 


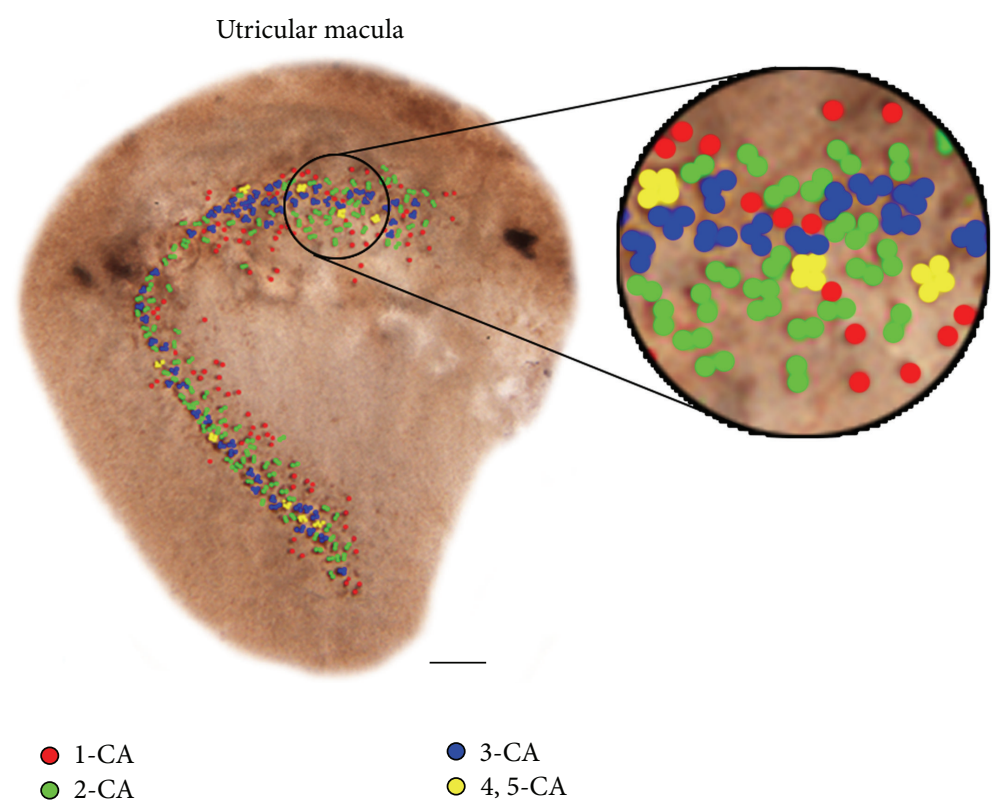

FiguRE 3: Utricular macula from the chinchilla showing the number and type of calretinin antibody-labeled calyx afferents. Calyx afferents are coded by the complexity of the afferent (red: simple calyx afferents, green: double complex calyx afferents, blue: triple complex calyx afferents, and yellow: quadruple and quintuple complex calyx afferents). Inset shows magnified calyx afferents coded by complexity.

\section{Conclusion}

Thermolysin is a proteolytic enzyme that digests specific protein bonds that anchor the otolithic membrane to macular sensory organs and extracellular matrix found within the stroma of cristae ampullares. Using thermolysin even in tissue that has been in fixative for up to two years can successfully create flattened mounts for the sensory epithelium, thereby facilitating visualization of the sensory organ and improving the accuracy of morphometric analysis.

\section{Conflict of Interests}

No financial disclosures or conflict of interests exists.

\section{References}

[1] J. Feder and J. M. Schuck, "Studies on the Bacillus subtilis neutral-protease- and Bacillus thermoproteolyticus thermolysin-catalyzed hydrolysis of dipeptide substrates," Biochemistry, vol. 9, no. 14, pp. 2784-2791, 1970.

[2] G. Allen, "Specific cleavage of the protein," in Laboratory Techniques in Biochemistry and Molecular Biology, G. Allen, Ed., Sequencing of Proteins and Peptides, chapter 3, pp. 43-71, Elsevier, Amsterdam, The Netherlands, 1981.

[3] H. Matsubara, A. Singer, R. Sasaki, and T. H. Jukes, "Observations on the specificity of a thermostable bacterial protease 'thermolysin"' Biochemical and Biophysical Research Communications, vol. 21, no. 3, pp. 242-247, 1965.

[4] H. Suzuki, Y. C. Lee, M. Tachibana, K. Hozawa, H. Wataya, and T. Takasaka, "Quantitative carbohydrate analyses of the tectorial and otoconial membranes of the guinea pig," Hearing Research, vol. 60 , no. 1, pp. 45-52, 1992.
[5] L. D. Saffer, R. Gu, and J. T. Corwin, "An RT-PCR analysis of mRNA for growth factor receptors in damaged and control sensory epithelia of rat utricles," Hearing Research, vol. 94, no. 1-2, pp. 14-23, 1996.

[6] L. Germain, M. Rouabhia, R. Guignard, L. Carrier, V. Bouvard, and F. A. Auger, "Improvement of human keratinocyte isolation and culture using thermolysin," Burns, vol. 19, no. 2, pp. 99-104, 1993.

[7] A. Gragnani, C. S. Sobral, and L. M. Ferreira, "Thermolysin in human cultured keratinocyte isolation," Brazilian Journal of Biology, vol. 67, no. 1, pp. 105-109, 2007.

[8] N. Perreault and J. Beaulieu, "Use of the dissociating enzyme thermolysin to generate viable human normal intestinal epithelial cell cultures," Experimental Cell Research, vol. 224, no. 2, pp. 354-364, 1996.

[9] C. Ripoll and G. Rebillard, "A simple technique to efficiently dissociate primary auditory neurons from 5 day-old rat cochleas," Journal of Neuroscience Methods, vol. 73, no. 2, pp. 123-128, 1997.

[10] J. Abouaish, M. Graham, P. Bansal-Pakala et al., "Successful isolation and transplantation of nonhuman primate islets using a novel purified enzyme blend," Transplantation, vol. 92, no. 8, pp. e40-e42, 2011.

[11] H. Suzuki, M. Furukawa, and T. Takasaka, "Quantitative uronic acid analysis of the otoconial membrane of the guinea pig," Hearing Research, vol. 114, no. 1-2, pp. 223-228, 1997.

[12] G. B. Wislocki and A. J. Ladman, "Selective and histochemical staining of the otolithic membranes, cupulae and tectorial membrane of the inner ear," Journal of Anatomy, vol. 89, no. 1, pp. 3-12, 1955.

[13] L. F. Bélanger, "Autoradiographic detection of S35 in the membranes of the inner ear of the rat," Science, vol. 118, no. 3070, pp. 520-521, 1953.

[14] L. F. Bélanger, "On the intimate composition of membranes of the inner ear," Science, vol. 123, no. 3207, pp. 1074-1075, 1956. 
[15] R. E. Shrader, L. C. Erway, and L. S. Hurley, "Mucopolysaccharide synthesis in the developing inner ear of manganese deficient and pallid mutant mice," Teratology, vol. 8, no. 3, pp. 257-266, 1973.

[16] M. Hultcrantz and D. Bagger-Sjöbäck, "Inner ear content of glycosaminoglycans as shown by monoclonal antibodies," Acta Oto-Laryngologica, vol. 116, no. 1, pp. 25-32, 1996. 

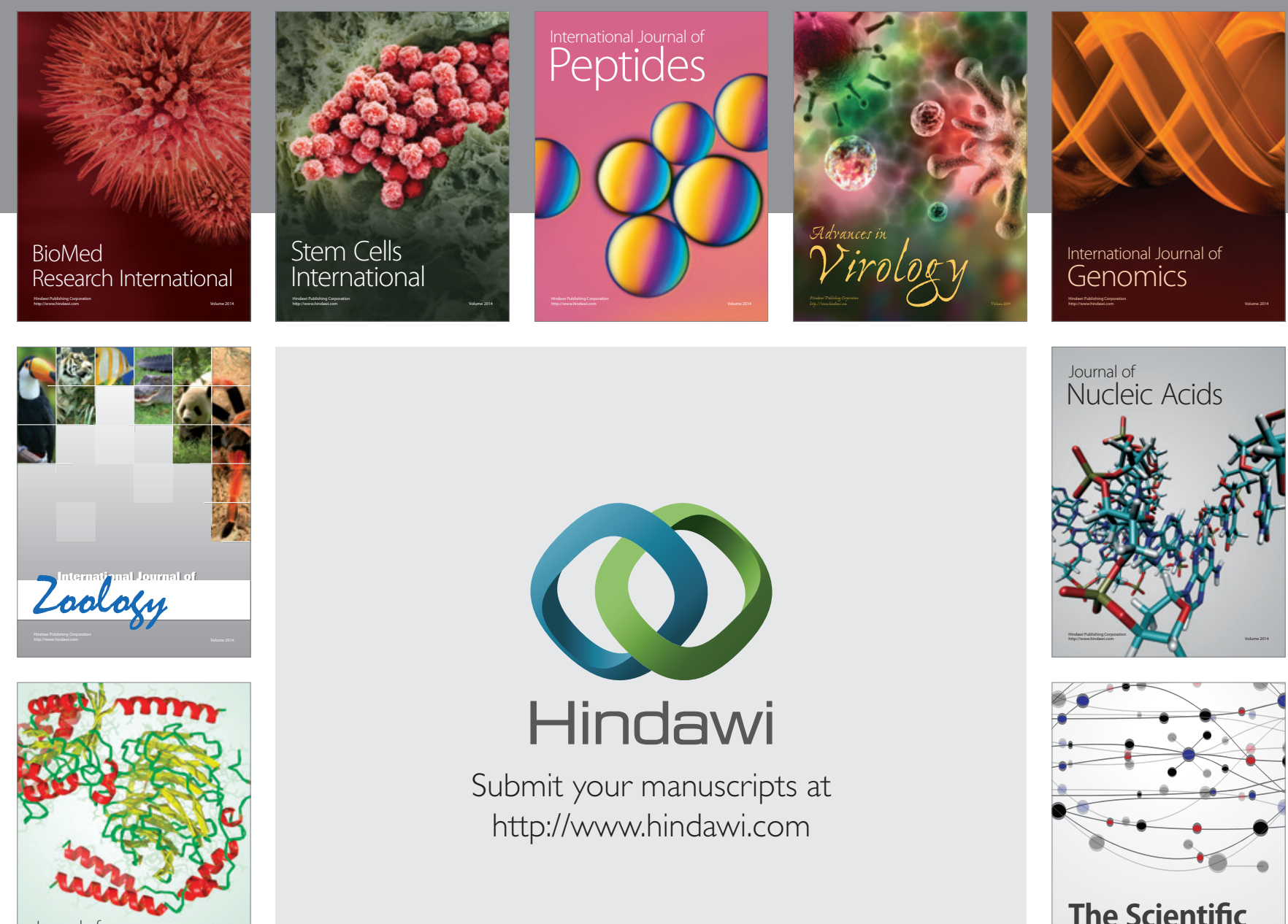

Submit your manuscripts at

http://www.hindawi.com

Journal of
Signal Transduction
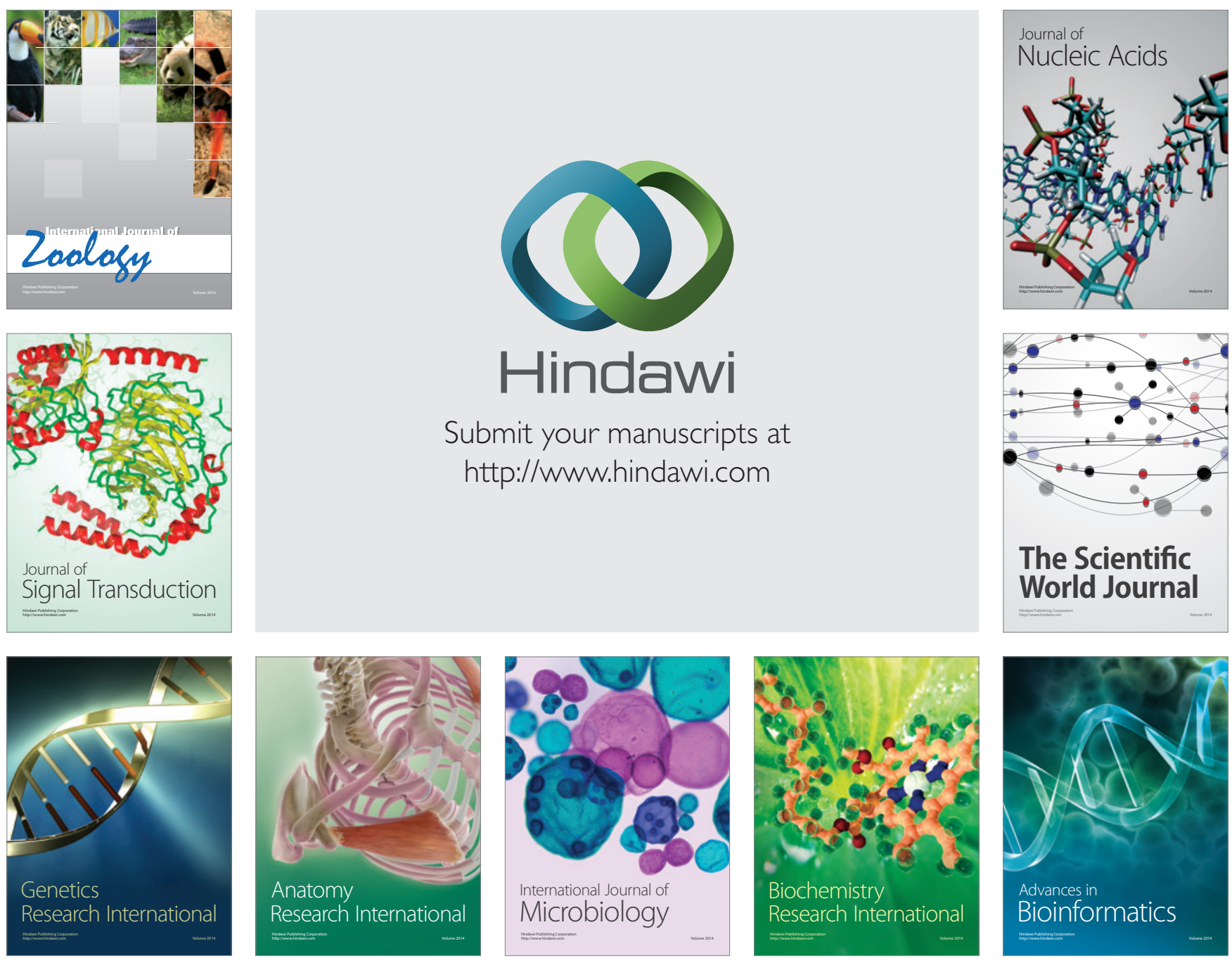

The Scientific World Journal
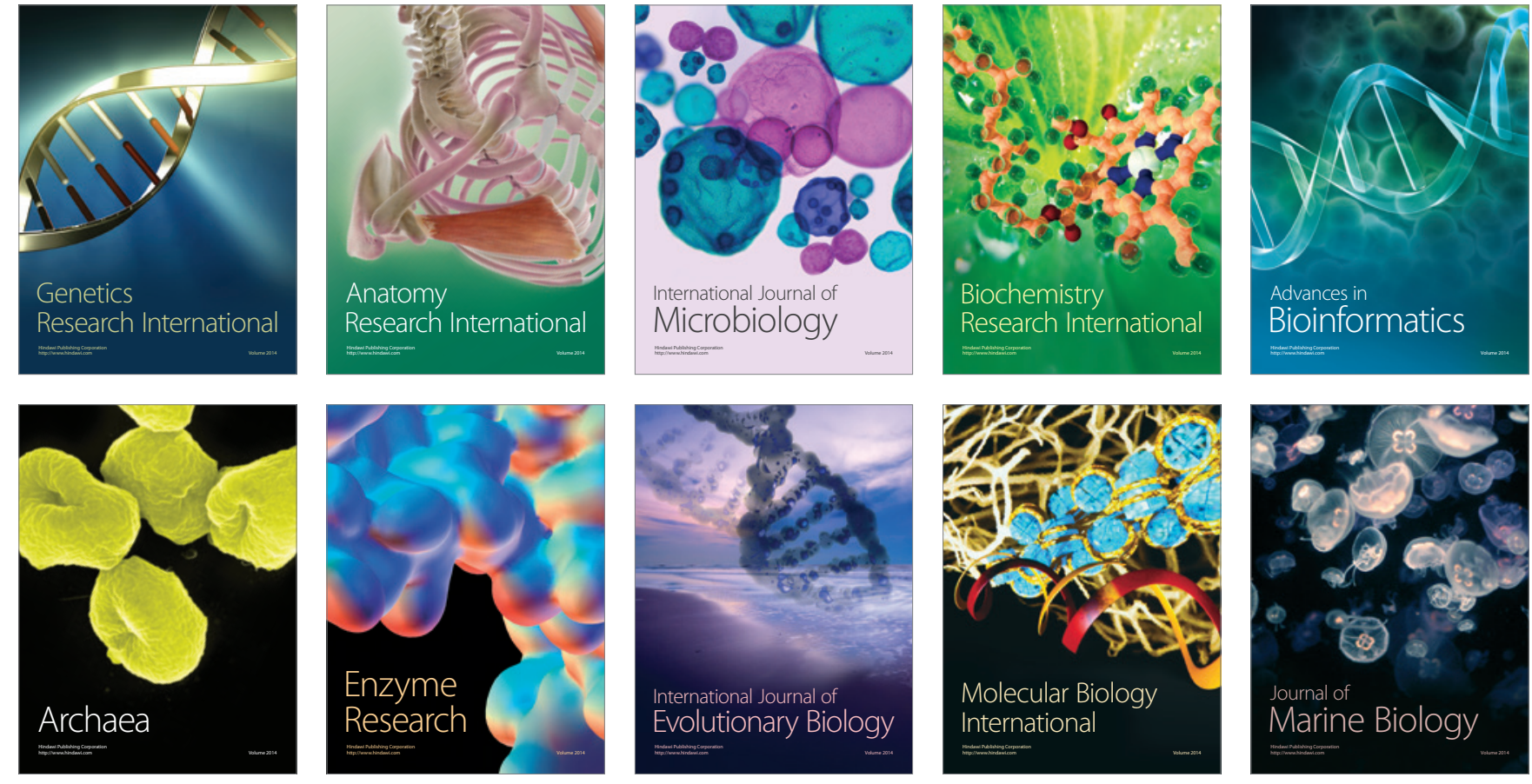